\title{
NONCOMPACT COMMUTATORS IN THE COMMUTANT OF A CYCLIC OPERATOR
}

\author{
JAMES D. STAFNEY
}

(Communicated by John B. Conway)

\begin{abstract}
We show that the commutant of the operator $S \oplus\left(I+S^{*}\right)$, where $S$ is the shift operator, contains two operators $A$ and $B$ such that $A B-B A$ is not compact operator.
\end{abstract}

Let $S$ be the shift operator and let $T=S \oplus\left(I+S^{*}\right)$. Herrero [1] has shown that $T$ and $T^{*}$ are cyclic and the commutant of $T$ is not commutative. In this paper we show that the commutant of $T$ fails to be commutative in the following stronger sense.

Theorem. The commutant of the operator $S \oplus\left(I+S^{*}\right)$ contains two operators $M, M_{1}$ such that $M M_{1}-M_{1} M$ is not a compact operator.

Proof. For convenience, let $W=S \oplus\left(I+S^{*}\right)$. So, $W$ is an operator (= bounded linear operator) on the Hilbert space $H=H^{2} \oplus H^{2}$. Each operator $A$ on $H$ can be expressed as a matrix

$$
A=\left(\begin{array}{ll}
A_{11} & A_{12} \\
A_{21} & A_{22}
\end{array}\right),
$$

where each $A_{i j} \in \mathscr{L}\left(H^{2}\right)$, the bounded linear operators on $H^{2}$. In particular,

$$
W=\left(\begin{array}{cc}
S & 0 \\
0 & I+S^{*}
\end{array}\right) .
$$

An easy computation shows that the operators

$$
E_{1}=\left(\begin{array}{ll}
I & 0 \\
0 & 0
\end{array}\right), \quad E_{2}=\left(\begin{array}{ll}
0 & 0 \\
C & 0
\end{array}\right)
$$

are in the commutant of $W$ provided that $X=C$ satisfies the operator equation

$$
\left(I+S^{*}\right) X=X S .
$$

Received by the editors July 1, 1988.

1980 Mathematics Subject Classification (1985 Revision). Primary 47B47.

Key words and phrases. Commutant, cyclic operator, commutator. 
Furthermore, a simple computation shows that

$$
E_{1} E_{2}-E_{2} E_{1}=\left(\begin{array}{cc}
0 & 0 \\
-C & 0
\end{array}\right) \text {. }
$$

These observations show that the theorem follows from Lemma 1.

Lemma 1. The operator equation (1) has a solution $X$ in $\mathscr{L}\left(H^{2}\right)$ which is not a compact operator.

To complete the proof of the theorem we now prove Lemma 1 based on the following lemmas and propositions. Let $T$ and $\varphi$ be as in Lemma 4. Let $A$ be the operator on $H^{2}$ with matrix $\left(a_{k, n}\right)$ corresponding to $\varphi$ as in Proposition 3. Then Proposition 3 and Lemma 4 show that $X=A$ is a solution (in $\mathscr{L}\left(H^{2}\right)$ ) of (1) which is not compact.

Proposition 2. The operator $X=A$ is a solution in $\mathscr{L}\left(H^{2}\right)$ of (1) if and only if the matrix $\left(a_{k, n}, k, n=0,1,2, \ldots\right.$, of $A$ relative to the orthonormal basis $u_{n}=e^{i n \theta}, n=0,1, \ldots$, satisfies:

(i) $a_{k, n}+a_{k+1, n}=a_{k, n+1}$;

(ii) the mairix $\left(a_{k, n}\right)$ determines a bounded operator on $l^{2}$.

Proof. Suppose $A$ is a solution of (1) and $\left(a_{k, n}\right)$ is its matrix. Then (ii) is clearly satisfied. To prove (i) we note

$$
\begin{aligned}
\sum_{k} a_{k, n+1} u_{k}=A u_{n+1}=A S u_{n} & =\left(I+S^{*}\right) A u_{n}=\left(I+S^{*}\right) \sum_{k} a_{k, n} u_{k} \\
& =\sum_{k} a_{k, n} u_{k}+\sum_{k} a_{k+1, n} u_{k} .
\end{aligned}
$$

The converse follows from similar reasoning.

Let $L^{2}$ denote $L^{2}(\mu)$ where $\mu$ is normalized Lebesgue measure on the unit circle. We regard $H^{2}$ as a subspace of $L^{2}$ whenever appropriate. For each $\varphi \in L^{2}$, we define the transformation $T_{\varphi}$ from $L^{2}$ into sequences by

$$
T_{\varphi} f(k)=\int\left(e^{i \theta}-1\right)^{k} \varphi\left(e^{i \theta}\right) f\left(e^{i \theta}\right) d \theta / 2 \pi,
$$

where $f \in L^{2}, k=0,1, \ldots$.

Proposition 3. For $\varphi \in L^{2}$, the matrix

$$
a_{k, n} \equiv \int\left(e^{i \theta}-1\right)^{k} e^{i n \theta} \varphi\left(e^{i \theta}\right) d \theta / 2 \pi
$$

is the matrix, relative to the orthonormal basis in Proposition 2, of a solution (compact solution) $X=A$ of (1) if and only if the restriction of $T_{\varphi}$ to $H^{2}$ determines a bounded (compact) linear transformation of $\mathrm{H}^{2}$ into $\mathrm{l}^{2}$.

Proof. If $\left(a_{k, n}\right)$ is the matrix of a bounded operator, then $T_{\varphi}$ clearly has the stated boundedness property. If $T_{\varphi}$ has the stated boundedness property, then 
$\left(a_{k, n}\right)$ is the matrix of an operator $A \in \mathscr{L}\left(H^{2}\right)$. An easy computation shows that $\left(a_{k, n}\right)$ satisfies (i) of Proposition 2. Thus, $X=A$ is a solution of (1).

Lemma 4. Let $T=T_{\varphi}$ where $\varphi$ is the characteristic function of the arc $\left\{e^{i \theta}: 0<\right.$ $\theta<\pi / 3\}$. Then

(i) $T$ is a bounded linear transformation of $L^{2}$ into $l^{2}$;

(ii) the restriction of $T$ to $H^{2}$ is not a compact operator.

Proof. For $f \in L^{2}$,

$$
T f(k)=\int_{0}^{\pi / 3}\left(e^{i \theta}-1\right)^{k} f\left(e^{i \theta}\right) d \theta / 2 \pi .
$$

If $b_{k}=\int_{0}^{1} x^{k} g(x) d x$, then since the Hilbert matrix is bounded,

$$
\sum_{k}\left|b_{k}\right|^{2} \leq C \int_{0}^{1}|g(x)|^{2} d x
$$

for some constant $C$. From this and a routine change of variable argument with $x=\left|e^{i \theta}-1\right|$, we conclude that $T$ is bounded.

We now prove (ii). For convenience, let $v(\theta)=e^{i \theta}-1$. A routine computation shows that

$$
T^{*} T f\left(e^{i \theta_{1}}\right)=\int_{0}^{\pi / 3}\left[1 /\left(1-\overline{v\left(\theta_{1}\right)} v(\theta)\right)\right] f(\theta) d \theta / 2 \pi
$$

for $f \in L^{2}$ and $0 \leq \theta_{1} \leq \pi / 3$; and, $T^{*} T f\left(e^{i \theta_{1}}\right)=0$, otherwise. We will show that the restriction of $T^{*} T$ to $H^{2}$ is not compact. For convenience, let $M$ denote the restriction of $T^{*} T$ to $H^{2}$. In particular, $M$ is a bounded linear transformation from $H^{2}$ into $L^{2}$. We will use Lemma 5 to show that $M$ is not compact.

Lemma 5. Suppose that $H, H^{\prime}$ are Hilbert spaces, that $A$ is a bounded linear transformation from $H$ into $H^{\prime}$ and that $x_{n} \in H$ for $n=1,2, \ldots$ such that

(i) $\left\|x_{n}\right\|=1$;

(ii) $\left(x_{m}, x_{n}\right) \rightarrow 0$ as $n \rightarrow \infty$ for each fixed $m$;

(iii) $\left\|A x_{n}\right\| \geq \delta>0$ for all $n$.

Then $A$ is not compact.

This lemma and Lemma 6 below show that $M$ is not compact, which completes the proof of (ii) in Lemma 4.

For $0<a<1$, let $z_{a}=(1+a) e^{i \pi / 3}$ and $f_{a}\left(e^{i \theta}\right)=\sqrt{a} /\left(e^{i \theta}-z_{a}\right)$. Clearly, each $f_{a} \in H^{2}$.

Lemma 6. There exist positive constants $C_{0}, C_{1}, C_{2}$ such that for $0<a$, $d<1$,

(i) $C_{0} \leq\left\|f_{a}\right\| \leq C_{1}$, 
(ii) $\left(f_{a}, f_{d}\right) \rightarrow 0$ as $d \rightarrow 0$ for each fixed $a$, and

(iii) $\left\|M f_{a}\right\| \geq C_{2}\left\|f_{a}\right\|$ where \|\| , ( , ) denote the norm and inner product on $L^{2}$.

Proof. Let $b=1+a(0<a<1)$ and let $I=\int_{-\pi}^{\pi} 1 /\left|e^{i \theta}-b\right|^{2} d \theta$. By noting that

$$
\left|e^{i \theta}-b\right|^{2}=a^{2}+b \theta^{2}-2 b R(\theta),
$$

where $R(\theta)=\cos \theta-\left(1-\theta^{2} / 2\right)$ one can show that

$$
\left(\frac{6}{11}\right)\left(\frac{1}{a}\right) \leq I \leq(3 \sqrt{2} \pi)\left(\frac{1}{a}\right) ;
$$

and, (i) easily follows.

To prove (ii) we note that $\left(f_{a}, f_{d}\right)$ is an integral over the interval $-\pi<\theta<\pi$ which is the sum of integrals over the subsets $J_{1}=\{\theta:|\theta-\pi / 3|<\delta\}$ and its complement $J_{2}$ in $[-\pi, \pi]$. By applying the Schwarz inequality to the integrals over each interval $J_{1}$ and $J_{2}$, by noting that $\int_{J_{1}}\left|f_{a}\right|^{2} d \theta \rightarrow 0$ as $\delta \rightarrow 0$ for $a$ fixed and by noting that $f_{d} \rightarrow 0$ as $d \rightarrow 0$ uniformly in $J_{2}$, we obtain (ii).

We now prove (iii). From the definition of $M$,

$$
M f_{a}\left(\theta_{1}\right)=\int_{0}^{\pi / 3}\left[1 /\left(1-\overline{v\left(\theta_{1}\right)} v(\theta)\right)\right] f_{a}(\theta) d \theta / 2 \pi
$$

for $0<\theta_{1}<\pi / 3$. For convenience, let $F=F\left(a, \theta_{1}, \theta\right)$ denote the integrand in the latter integral. It is easily seen that

1. the argument of $f_{a}(\theta)$ varies over an interval of length $\leq \pi / 2$ for $0<$ $a<1$ and $0 \leq \theta \leq \pi / 3$.

A geometric argument shows that $\overline{v\left(\theta_{1}\right)} v(\theta)$ lies in the intersection of two circles both of radius one and with centers at $e^{i \pi / 3}$ and $e^{-i \pi / 3}$ for $0 \leq \theta$, $\theta_{1} \leq \pi / 3$. From this we conclude that

2. the arugment of $1-\overline{v\left(\theta_{1}\right)} v(\theta)$ varies over an interval of length $\leq \pi / 3$ for $0 \leq \theta, \theta_{1} \leq \pi / 3$; and, for some $C>0$,

$$
\left|1-\overline{v\left(\theta_{1}\right)} v(\theta)\right| \leq C\left(1-\overline{\mid v\left(\theta_{1}\right)} v(\theta) \mid\right), \quad 0 \leq \theta, \theta_{1} \leq \pi / 3 .
$$

Thus, in particular, the argument of $F$ varies over an interval of length $\leq 5 \pi / 6$ for $0<a<1$ and $0 \leq \theta, \theta_{1} \leq \pi / 3$. So, for some complex number $\lambda$ with $|\lambda|=1$, the argument of $\lambda F$ lies in the interval $[-5 \pi / 12,5 \pi / 12]$. Therefore,

$$
\left|M f_{a}\left(\theta_{1}\right)\right| \geq C \int_{0}^{\pi / 3}\left|F\left(a, \theta_{1}, \theta\right)\right| d \theta, \quad 0<\theta_{1}<\pi / 3
$$

for some $C>0$.

Let $t=|v(\theta)|$ and $s=\left|v\left(\theta_{1}\right)\right|$. From (2) and the definition of $f_{a}$ it follows that

$$
|F| \geq C(1 /(1-s t))(1 / \sqrt{a}), \quad 0<s<1,1-a<t<1,
$$


for some $C>0$. By a change of variable we conclude that for some $C>0$,

$$
\left|M f_{a}\left(\theta_{1}\right)\right| \geq(C / \sqrt{a}) \int_{1-a}^{1} 1 /(1-s t) d t \geq(C / \sqrt{a}) \log (a / 2(1-s)) \geq 0
$$

for $s>1-a / 2$. Thus, for some $C>0, C$ independent of $a$, we have

$\int_{0}^{\pi / 3}\left|M f_{a}\left(\theta_{1}\right)\right|^{2} d \theta_{1} \geq(C / a) \int_{1-a / 2}^{1}[\log (a / 2(1-s))]^{2} d s=(C / 2) \int_{0}^{1}(\log t)^{2} d t$, which, together with (i), proves (ii).

\section{ACKNOWLEDGMENT}

The referee has pointed out that the operator $A=S^{*} \otimes I$ is cyclic and the commutant of $A$ contains operators $B$ and $C$ such that $B C-C B$ is not compact. However, $A^{*}$ is not cyclic. So, the significance of Herrero's example is that both $T$ and $T^{*}$ are cyclic. The referee has also noted that if $A$ denotes the infinite direct sum of the operators $(1 / n) I+10^{-n} T, n=1,2, \ldots$, then $A$ and $A^{*}$ are cyclic and the commutant of $A$ contains a pair of operators with noncompact commutator. The point of our theorem is that it is not so clear that the operator $T$ has this property. Because of the referees comments, we changed our original abstract and first paragraph.

\section{REFERENCES}

1. D. A. Herrero, On multicyclic operators, Integral Equations Operator Theory 1 (1978), pp. 57, 102.

University of California, Department of Mathematics \& Computer Science, RiverSIDE, CALIFORNIA 92521 